The Astrophysical JouRnal, 550:65-74, 2001 March 20

(C) 2001. The American Astronomical Society. All rights reserved. Printed in U.S.A.

\title{
SUPERMASSIVE BLACK HOLES IN BULGES ${ }^{1}$
}

\author{
Marc Sarzi, ${ }^{2,3}$ Hans-Walter Rix, ${ }^{3}$ Joseph C. Shields, ${ }^{4}$ Greg Rudnick, ${ }^{3,5}$ Luis C. Ho, ${ }^{6}$ Daniel H. McIntosh, ${ }^{5}$ \\ Alexei V. FilipPenko, ${ }^{7}$ and Wallace L. W. Sargent ${ }^{8}$ \\ Received 2000 August 1; accepted 2000 November 9
}

\begin{abstract}
We present spatially extended gas kinematics at parsec-scale resolution for the nuclear regions of four nearby disk galaxies and model them as rotation of a gas disk in the joint potential of the stellar bulge and a putative central black hole. The targets were selected from a larger set of long-slit spectra obtained with the Hubble Space Telescope as part of the Survey of Nearby Nuclei with STIS. They represent the four galaxies (of 24) that display symmetric gas velocity curves consistent with a rotating disk. We derive the stellar mass distribution from the STIS acquisition images adopting the stellar mass-to-light ratio normalized to match ground-based velocity dispersion measurements over a large aperture. Subsequently, we constrain the mass of a putative black hole by matching the gas rotation curve, following two distinct approaches. In the most general case we explore all the possible disk orientations; alternatively, we constrain the gas disk orientation from the dust-lane morphology at similar radii. In the latter case the kinematic data indicate the presence of a central black hole for three of the four objects, with masses of $10^{7}-10^{8} M_{\odot}$, representing up to $\sim 0.025 \%$ of the host bulge mass. For one object (NGC 2787) the kinematic data alone provide clear evidence for the presence of a central black hole even without external constraints on the disk orientation. These results illustrate directly the need to determine black hole masses by differing methods for a large number of objects, demonstrate that the variance in black hole/bulge mass is much larger than previously claimed, and reinforce the recent finding that the black hole mass is tightly correlated with the bulge stellar velocity dispersion $\sigma$.

Subject headings: black hole physics - galaxies: kinematics and dynamics — galaxies: nuclei
\end{abstract}

\section{INTRODUCTION}

Over the last few years evidence has mounted that supermassive black holes (SMBHs) are nearly ubiquitous in galactic centers. This finding has resulted from highresolution observations of various kinematical tracers of the central gravitational potential, such as stars, ionized gas, or water masers (see, e.g., Ho 1999 for a review). Additional support for this picture is provided by spectroscopic surveys demonstrating that weak active galactic nuclei (AGN) activity is common in nearby galaxies, especially among earlytype systems (E-Sb; Ho, Filippenko, \& Sargent 1997a and references therein). An important fraction of these nuclei further exhibit broad-line emission and/or compact X-ray or radio sources that are the hallmarks of quasar activity, presumably powered by accretion onto a black hole (e.g., Ho et al. 1997b; Terashima, Ho, \& Ptak 2000; Nagar et al. 2000).

If SMBHs are indeed an integral part of galaxies and their formation a natural question is whether the black hole

\footnotetext{
${ }^{1}$ Based on observations with the Hubble Space Telescope obtained at STScI, which is operated by AURA, Inc., under NASA contract NAS 5-26555.

2 Dipartimento di Astronomia, Università di Padova, Vicolo dell'Osservatorio 5, I-35122 Padova, Italy.

${ }^{3}$ Max-Planck-Institut für Astronomie, Königstuhl 17, Heidelberg D69117, Germany; rix@mpia-hd.mpg.de.

4 Physics and Astronomy Department, Ohio University, Athens, $\mathrm{OH}$ 45701; shields@phy.ohiou.edu.

${ }^{5}$ Steward Observatory, University of Arizona, Tucson, AZ 85721; dmac,grudnick@as.arizona.edu.

6 The Observatories of the Carnegie Institution of Washington, 813 Santa Barbara Street, Pasadena, CA 91101-1292; 1ho@ociw.edu.

7 Astronomy Department, University of California, Berkeley, CA 94720-3411; alex@astro.berkeley.edu.

${ }^{8}$ Palomar Observatory, Caltech 105-24, Pasadena, CA 91125; wws@astro.caltech.edu.
}

mass $M_{\mathrm{BH}}$ is related to larger properties of the host galaxy. A correlation between $M_{\mathrm{BH}}$ and stellar mass of the spheroidal component $M_{\text {bulge }}$ was suggested by Kormendy (1993) and later quantified by Kormendy \& Richstone (1995), Magorrian et al. (1998), and Ho (1999). As investigated recently by Kauffmann \& Haehnelt (2000), such a correlation might arise naturally in hierarchical scenarios for galaxy formation, and is expected if local SMBHs are the dormant relics of past quasar activity. Evidence of a close connection between galaxy structure and SMBHs has been bolstered by recent reports of a correlation between $M_{\mathrm{BH}}$ and the velocity dispersion of the surrounding stellar bulge (Ferrarese \& Merritt 2000, hereafter FM00; Gebhardt et al. 2000a, hereafter G00).

The largest compilation of SMBH mass estimates obtained with a single technique, namely, axisymmetric two-integral models for the central stellar kinematics, is that of Magorrian et al. (1998). For most of the 32 early-type galaxies of their sample, they found that Hubble Space Telescope (HST) photometric and ground-based kinematical data were consistent with the presence of a central supermassive black hole. Based on their data they claim that $M_{\mathrm{BH}} \simeq 0.006 M_{\text {bulge }}$ with a scatter of only a factor of $\sim 3$. While the restrictive orbital assumptions in their modeling can lead to an overestimate of the black hole mass (Magorrian et al. 1998), their high detection rate nonetheless underscores the apparent prevalence of SMBHs and their close connection to the surrounding stellar bulge.

Despite this recent progress, the demography of SMBHs is far from complete. Most of the reported SMBHs have been detected in bulge-dominated systems, and it is not clear if the $M_{\mathrm{BH}} / M_{\text {bulge }}$ relation holds also in the case of disk-dominated galaxies. This issue is important in light of theories for bulge formation via secular evolution of disk structures (e.g., Norman, Sellwood, \& Hasan 1996). In addi- 
tion, it is still not fully understood whether the apparent lack of low-mass central black holes in very massive galaxies is because of selection effects, in which case the correlated distribution of points in the $M_{\mathrm{BH}} / M_{\text {bulge }}$ plane actually represents only an upper envelope. In Seyfert nuclei and quasars, measurements of $M_{\mathrm{BH}}$ derived from reverberation mapping experiments provide intriguing evidence that the average $M_{\mathrm{BH}} / M_{\text {bulge }}$ ratio in active galaxies is systematically lower than in normal ones (Ho 1999; Wandel 1999; Kaspi et al. 2000; Gebhardt et al. 2000b), even though this discrepancy may disappear in the $M_{\mathrm{BH}} / \sigma$ ratio (Gebhardt et al. $2000 \mathrm{~b}$ ). Independent determinations of $M_{\mathrm{BH}}$ in active galaxies are desirable for confirmation of these results. Black hole mass estimates and the corresponding Eddington luminosity $L_{\mathrm{Edd}}$ are also of fundamental importance for testing theories of accretion physics in AGNs, since models typically predict a strong dependence of accretion disk structure on $\dot{M} / M_{\mathrm{BH}}$, or alternatively $L / L_{\mathrm{Edd}}$ (e.g., Narayan, Mahadevan, \& Quataert 1998).

In order to address these and other issues we initiated a Survey of Nearby Nuclei with STIS (SUNNS) on HST, with kinematic information obtained from measurements of nebular emission lines. Because of its collisional nature, gas promises a conceptually easier way to trace the gravitational potential of a galaxy nucleus than stars, because nearly circular orbits can be assumed; at the same time, however, gas is more susceptible to nongravitational forces and may not be in equilibrium. Use of emission-line tracers to constrain a central mass concentration is thus limited to cases where strong evidence exists that the gas dynamics are dominated by gravity.

The paper is organized as follows. In $\S 1$ we present the spectroscopic and photometric STIS observations, and in $\S 2$ we describe our modeling of the ionized gas kinematics. We present our results in $\S 3$ and draw our conclusions in $\S 4$.

\section{OBSERVATIONS AND DATA REDUCTION}

In carrying out SUNNS, long-slit spectra were obtained with STIS for the central regions of 24 nearby, weakly active galaxy nuclei (Filippenko \& Sargent 1985; Ho et al. 1997a). Full details of the observations and data calibration are reported by H. W. Rix et al. (2001, in preparation). The observations were acquired in 1998 and 1999, with the 0.2 $\times 52^{\prime \prime}$ slit placed across each nucleus along an operationally determined position angle (P.A.), which is effectively random with respect to the projected major axis of any galaxy subsystem. After initial $20 \mathrm{~s}$ acquisition expo- sures with the long pass filter (roughly equivalent to $R$ ), from which we derive surface photometry of the nuclei, two spectral exposures totaling $\sim 30$ minutes and three exposures totaling $\sim 45$ minutes were obtained with the G430L and G750M gratings, respectively. The resulting spectra span $3300-5700 \AA$ and $630-6850 \AA$ with spectral resolution for extended sources of 10.9 and $2.2 \AA$, respectively. The telescope was offset by 0 0.05 ( $\approx 1$ pixel) along the slit direction between repeated exposures to aid in the removal of hot pixels.

The two-dimensional spectra were bias and dark subtracted, flat fielded, aligned, and combined to single blue and red spectra, cleaned of remaining cosmic rays and hot pixels, and corrected for geometrical distortion. The data were wavelength and flux calibrated with standard STSDAS procedures. The exact reduction steps differed slightly from image to image, in particular the image combining, dithering, and hot pixel removal. A detailed discussion of the reduction steps and the two-dimensional spectra will appear in H. W. Rix et al. (2001, in preparation). For the kinematic analysis reported here, we used only the red, high-resolution spectra. We determined the radial velocity of the ionized gas as a function of position along the slit from Doppler shifts of the $\mathrm{H} \alpha$ and $[\mathrm{N} \mathrm{II}] \lambda \lambda 6548$, 6583 emission lines. Measurement of these features was carried out using the program SPECFIT as implemented in IRAF (Kriss 1994), assuming a common velocity frame and Gaussian profile for the three lines and a $[\mathrm{N} \mathrm{II}]$ doublet ratio (1:3) dictated by atomic parameters. SPECFIT employs $\chi^{2}$-minimization methods that provide error estimates for the measured velocities.

Of the target galaxies, 12 displayed spatially resolved nebular emission at a level that was detectable in our data. For these sources, many of the measured gas velocity profiles showed distinct asymmetries and other features that appear inconsistent with steady-state orbital motion. We consequently selected for further detailed study only those objects for which the gas velocity curves were extended, single valued, and nearly reflection-symmetric about the center of the galaxy; six objects met these criteria. We subsequently eliminated two of the remaining objects (NGC 3351 and NGC 4548) from the present study since surface photometry indicates that their central regions exhibit large deviations from sphericity or strong isophote twisting; we defer discussion of these sources to a later analysis. The remaining four objects that we present here are NGC 2787, NGC 4203, NGC 4459, and NGC 4596. Global parameters for these galaxies are given in Table 1, while their surface

TABLE 1

Basic Parameters of Selected Galaxies

\begin{tabular}{|c|c|c|c|c|c|c|c|c|c|}
\hline $\begin{array}{l}\text { Galaxy } \\
\text { (1) }\end{array}$ & $\begin{array}{l}\text { Morphological Type } \\
\text { (2) }\end{array}$ & $\begin{array}{l}B_{\text {tot }} \\
(3)\end{array}$ & $\begin{array}{c}i \\
(4)\end{array}$ & $\begin{array}{l}\text { P.A. } \\
\text { (5) }\end{array}$ & $\begin{array}{c}\text { Nuclear Type } \\
\text { (6) }\end{array}$ & $\begin{array}{l}\text { Distance } \\
\text { (7) }\end{array}$ & $\begin{array}{c}\sigma \\
(8)\end{array}$ & $\begin{array}{l}\text { STIS Slit P.A. } \\
\text { (9) }\end{array}$ & $\begin{array}{l}\text { Date } \\
(10)\end{array}$ \\
\hline NGC $2787 \ldots \ldots$ & $\mathrm{SB}(\mathrm{r}) 0+$ & 11.82 & 50 & 117 & L1.9 & 13.0 & 210 & 33.2 & 1998 Dec 5 \\
\hline NGC $4203 . . . .$. & $\mathrm{SAB} 0-$ : & 11.80 & 21 & 10 & L1.9 & 9.7 & 124 & 105.3 & 1999 Apr 18 \\
\hline NGC $4459 . . . .$. & $\mathrm{SA}(\mathrm{r}) 0+$ & 11.32 & 41 & 110 & $\mathrm{~T} 2:$ & 16.8 & 189 & 92.9 & 1999 Apr 23 \\
\hline NGC $4596 \ldots . .$. & $\mathrm{SB}(\mathrm{r}) 0+$ & 11.35 & 42 & 135 & L2:: & 16.8 & 154 & 70.3 & 1998 Dec 20 \\
\hline
\end{tabular}

Note.-Cols. (2), (3), (4), and (5): morphological type, total apparent magnitude $B_{\text {tot }}$, inclination, and major axis P.A. from de Vaucouleurs et al. 1991. Col. (6): nuclear emission type from Ho et al. 1997a: $\mathrm{L}=$ LINER, and $\mathrm{T}=$ transition object. The number attached to the class letter designates the type, while the ":" and "::" indicate an uncertain or highly uncertain classification, respectively. Col. (7): distance from Tully 1988 in Mpc. Col. (8): Ground-based central stellar velocity dispersion $\sigma$ in $\mathrm{km} \mathrm{s}^{-1}$, taken from Dalle Ore et al. 1991 for the first three sources and from Kent 1990 for NGC 4596; these authors obtained measurements through apertures of size 1 1.5 5 4."0 and 1".5 $\times 5$ 5.4, respectively. Col. (9): P.A. of the STIS aperture. Col. (10): UT observation date. 


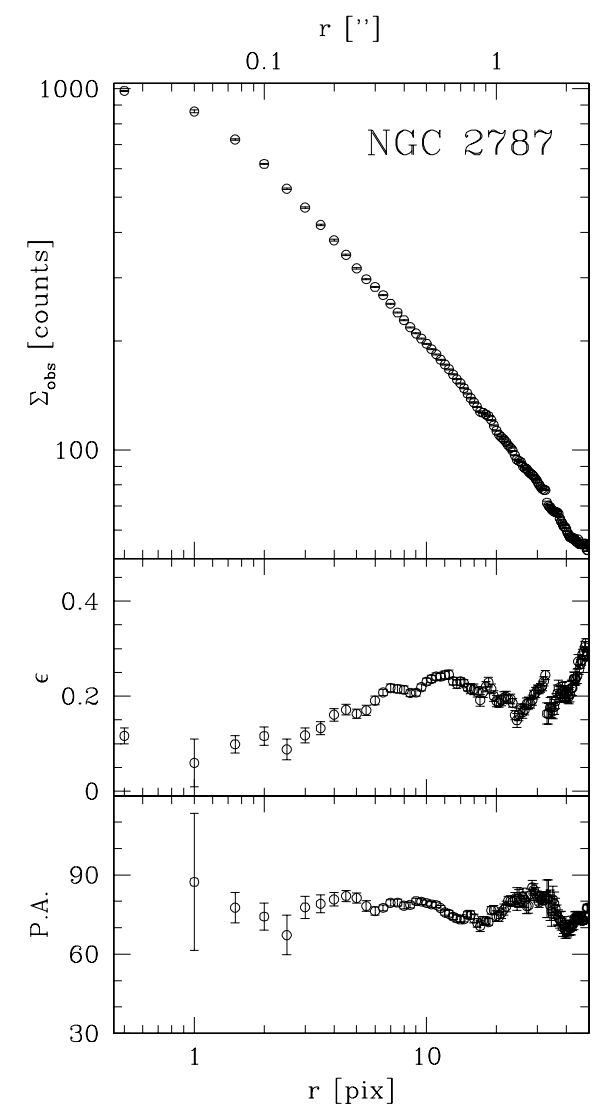

FIG. $1 a$

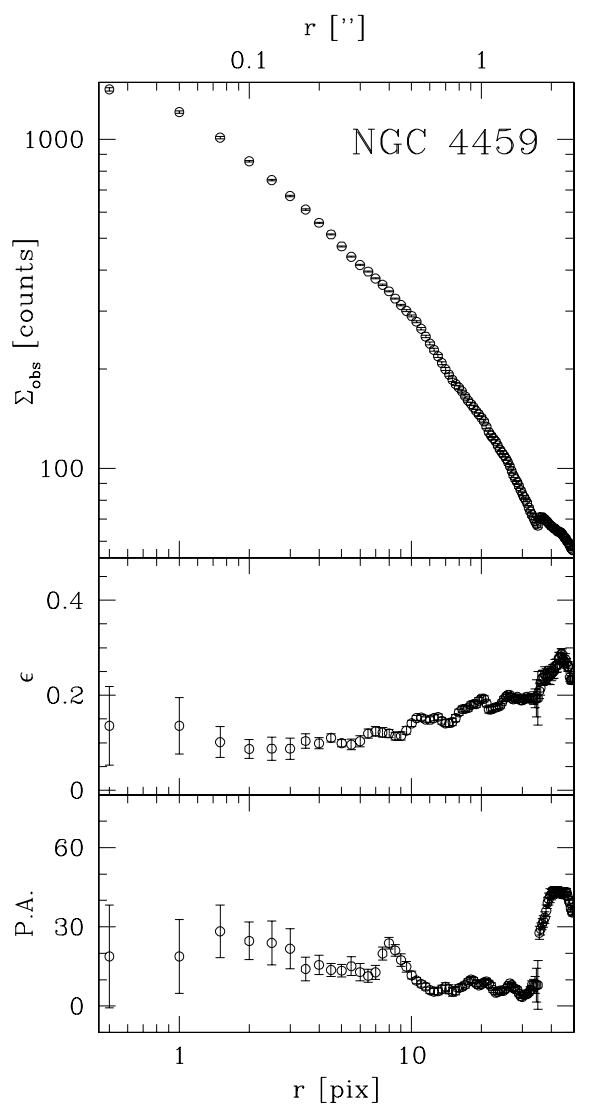

FIG. $1 c$

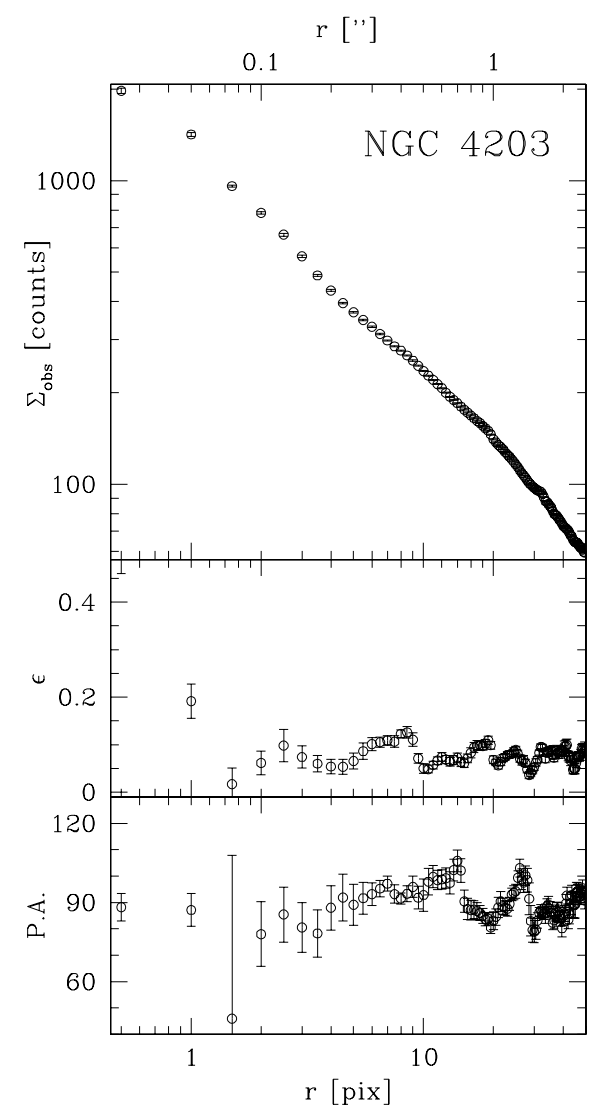

FIG. $1 b$

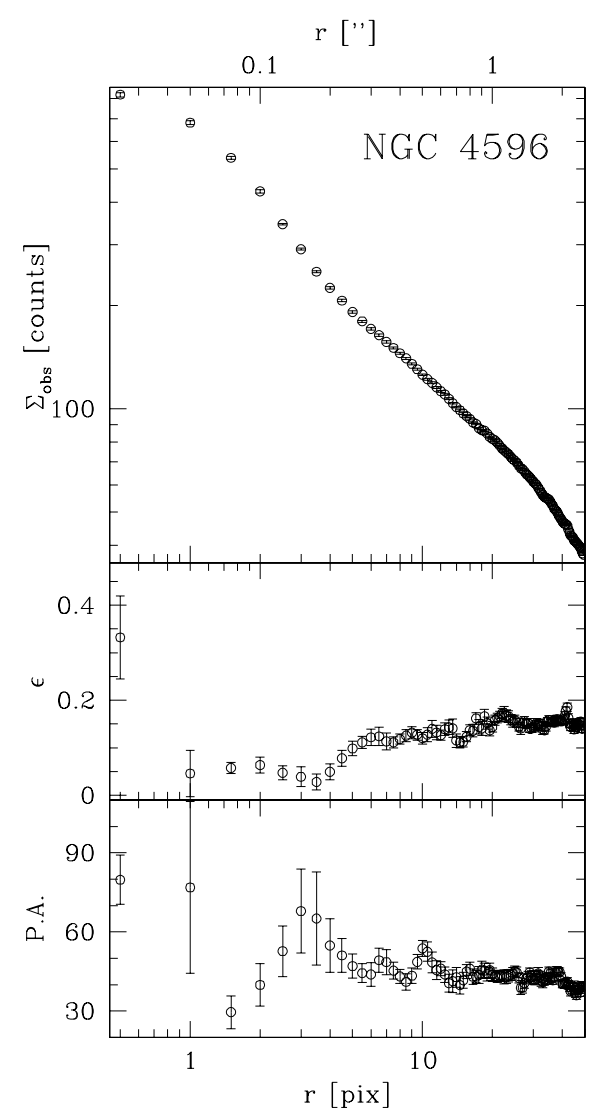

Fig. $1 d$

FIG. 1.-Radial profiles of the surface brightness, ellipticity, and P.A. in NGC 2787, NGC 4203, NGC 4459, and NGC 4596 obtained from the STIS broadband acquisition images. 
brightness, ellipticity, and position angle profiles are shown in Figure 1. These radial profiles were obtained with the task ELLIPSE in IRAF, after masking the most prominent dust features (e.g., NGC 2787). We adopted ellipses with linearly spaced semimajor axes, with a minimum radius of 0.5 pixel.

\section{THE MODELING OF THE IONIZED GAS KINEMATICS}

We used the data to derive constraints on the presence and mass of a SMBH by modeling the gas kinematics in the combined gravitational potential of the stars and of a central dark mass. The projected light distribution in the centers of these galaxies is relatively round (axis ratio $b / a \geq 0.7$ ), and we consequently assumed spherical symmetry for the stellar mass distribution. This approximation is sufficient to roughly estimate the stellar mass-to-light ratio within $\lesssim 3^{\prime \prime}$. We further assumed that the circumnuclear ionized gas is moving on planar, closed, and hence nearly circular orbits in the total gravitational potential. The spatial gas distribution was taken to be a disk, whose orientation is specified by its inclination $i$ with respect to the sky plane, and by the angle $\phi$ between the directions of its projected kinematic major axis and the slit position. To derive the stellar portion of the gravitational potential and to make predictions for the gas velocities, we proceeded in several steps, as follows.

\subsection{Stellar Mass Profile}

We derived the deprojected stellar luminosity density $v(r)$ from the observed surface brightness profile $\Sigma_{\text {obs }}(R)$ in the STIS acquisition image. The intrinsic surface brightness profile $\Sigma(R)$ was modeled as a sum of Gaussian components (Monnet, Bacon, \& Emsellen 1992), which were convolved with the HST point-spread function (PSF). The PSF itself was also represented as a sum of Gaussians and was derived from stars present in the STIS acquisition images. This yielded a PSF very similar to the synthetic ones obtained using the Tiny Tim package (Krist \& Hook 1999) and with stellar spectral energy distributions. The convolved model was then compared with $\Sigma_{\text {obs }}(R)$ in order to determine the optimal scaling coefficients for the Gaussian components. In practice, we constrained the Gaussian width coefficients, $\sigma_{i}$, to be a set of logarithmically spaced values, thus sim- plifying the multi-Gaussian decomposition into a general nonnegative linear least-squares problem for the corresponding Gaussian amplitudes, $\alpha_{i}$. The resulting deconvolved $\Sigma(R)$ is then readily deprojected to obtain $v(r)$. For a radially constant mass-to-light ratio $\Upsilon$, a multi-Gaussian description also results for the stellar mass density $\rho(r)=\Upsilon v(r)$, whose contribution $\Phi_{*}(r)$ to the total potential $\Phi(r)$ can be computed conveniently in terms of error functions.

In applying this method to NGC 2787, NGC 4203, and NGC 4596, we found a pointlike, presumably nonstellar light component in addition to the extended stellar light. We note that the absence of such a component in NGC 4459 is consistent with its comparatively weak indications of AGN activity, reflected in its spectroscopic classification as an $\mathrm{H}$ II/LINER transition object. Central point sources manifest themselves by abrupt changes in the surface brightness profile slope in the innermost 1-2 pixels and are well fit by PSF-convolved point sources. We consequently removed this component from the measured $\Sigma_{\text {obs }}(R)$ in order to measure only stellar light. As an example, in Figure 2 we show the multi-Gaussian fit to $\Sigma_{\text {obs }}(R)$ for NGC 4203, along with the recovered luminosity density profile $v(r)$ and the shape of the corresponding circular velocity curve $V_{c}(r)$.

\subsection{Stellar Mass-to-Light Ratio}

Values of $\Upsilon$ can be constrained dynamically using measurements of the stellar velocity dispersion $\sigma(r)$ averaged over a size scale sufficiently large that a plausible SMBH will not dominate the potential. We used ground-based measurements through apertures of several square arcseconds for this purpose (Table 1). We assumed a constant $\Upsilon$ for each galaxy and solved the Jeans equation in the spherical isotropic case (Binney \& Tremaine 1987) for the velocity dispersion profile $\sigma(r)$. We then integrated along the line of sight to obtain the projected $\sigma_{p}(R)$ and computed a seeing-convolved, surface-brightness-weighted mean $\left\langle\sigma_{p}(R)\right\rangle$ within the given aperture. Finally, we adjusted $\Upsilon$ until $\left\langle\sigma_{p}(R)\right\rangle$ matched the observed $\sigma$.

As a refining step, we included the effect of a central black hole on $\left\langle\sigma_{p}(R)\right\rangle$ by producing a $\Upsilon\left(M_{\mathrm{BH}}\right)$ curve, which is
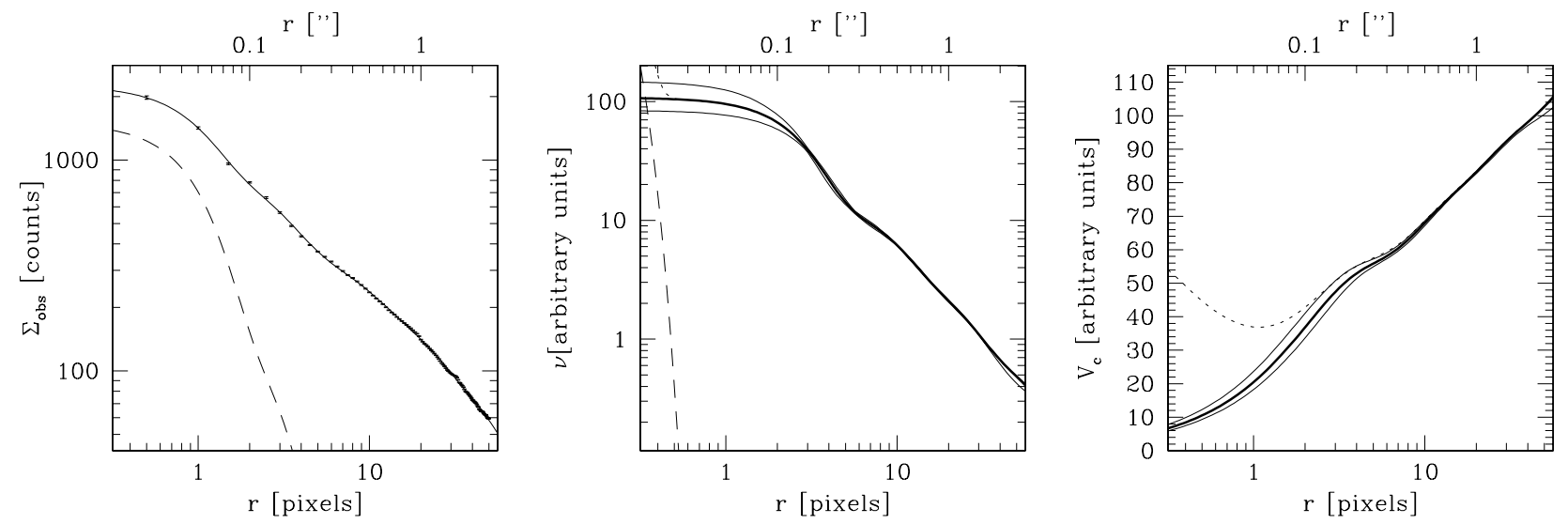

FIG. 2.-Deprojection steps for the stellar mass profile of NGC 4203: Left: Multi-Gaussian fit to the observed surface brightness $\Sigma_{\text {obs }}(R)$ of NGC 4203 (solid line); the inferred point-source contribution to the profile is also shown (dashed line). Center: Recovered intrinsic stellar luminosity profile $v(r)$ (thick solid line) after removal of the point source, and $3 \sigma$ confidence limits on $v(r)$ (thin solid lines) obtained deprojecting different Monte Carlo realizations of $\Sigma_{\text {obs }}(R)$. Inclusion of this compact source (dashed curve) reproducing the observations in their entirety would produce a narrow spike at the center of $v(r)$ (dotted line). Right: Circular velocity profile $V_{c}(r)$ (thick solid line) that results if the mass density is proportional to $v(r)$, along with $3 \sigma$ confidence curves $($ thin solid line). The dotted line shows the effect of assigning mass density proportional to the luminosity density profile that includes the central compact feature. 

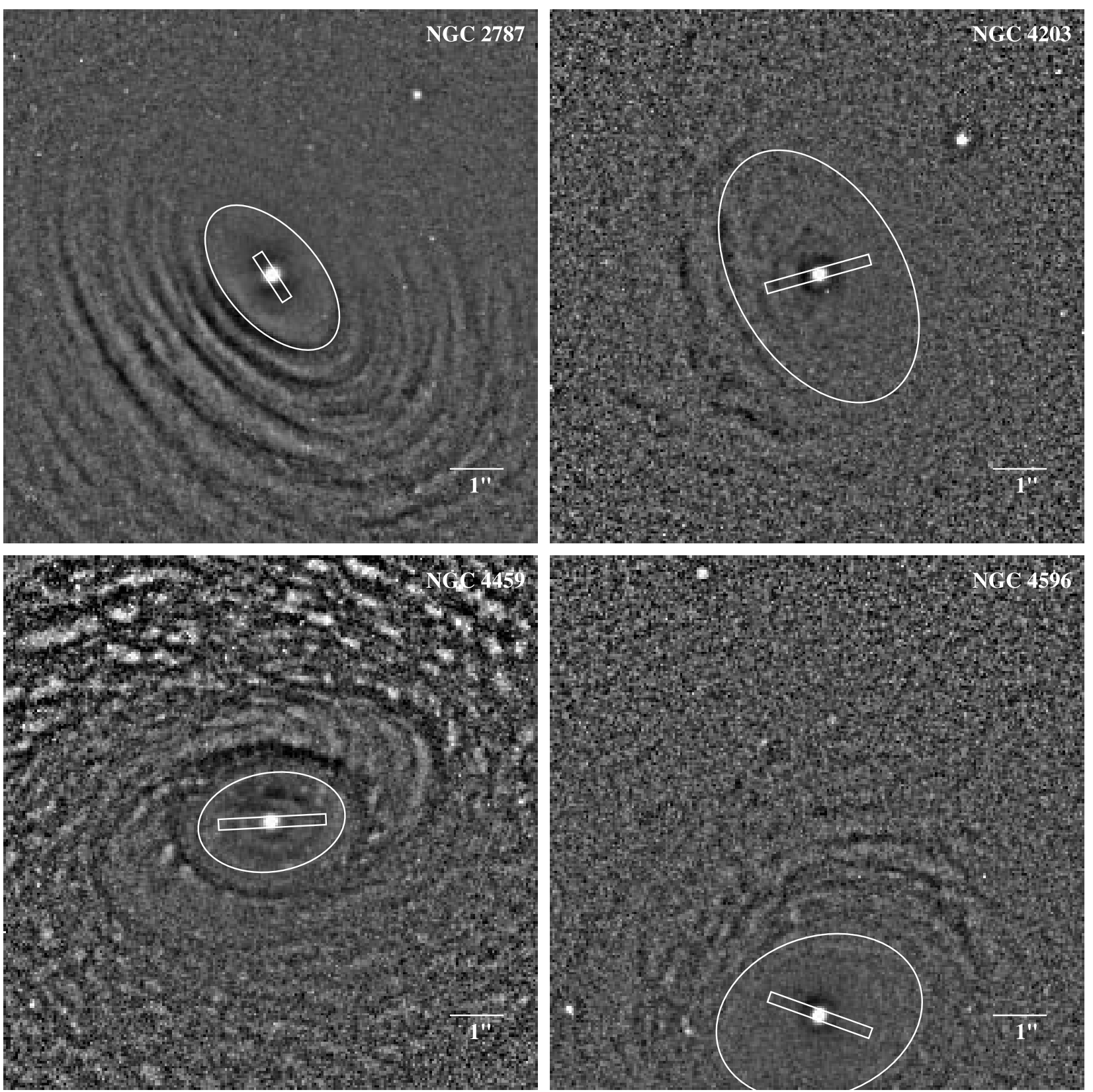

FIG. 3.-The $10^{\prime \prime} \times 10^{\prime \prime}$ unsharp-masked WFPC2 images for the central regions of our modeled galaxies. North is up, east is to the left. Superposed on each frame is the ellipse showing the assumed gaseous disk orientation and the STIS slit aperture, where the depicted length corresponds to the maximum extent of our kinematic measurements. All images were derived from frames obtained in the F555W passband, except for NGC 4596, for which the F606W filter was used.

monotonically decreasing with increasing black hole mass, from the $\Upsilon(0)$ maximum value without any central mass concentration down to the limiting zero value corresponding to some maximum allowed black hole mass (typically $\left.\sim 10^{9} M_{\odot}\right)$.

All the model steps, including the multi-Gaussian fit of $\Sigma(R)$, recovery of $v(r)$, calculation of $V_{c}(r)$, and reconstruction of $\sigma(r)$ and $\sigma_{p}(R)$, were tested and verified using Hernquist (1990) analytical models for all these functions.

\subsection{Gas Velocity Field}

The prediction of the observed gas velocity field for our galaxies depends on the orientation of the gas disk in each case. We cannot expect that the parsec-scale central gas disk is coplanar with the vastly larger stellar galaxy disk; indeed, there are examples where this is clearly not the case (e.g., NGC 3227; Schinnerer et al. 2000). We therefore modeled the gas kinematics with the disk inclination angles $i$ and $\phi$ for the gas initially unconstrained.

Inspection of acquisition and archival WFPC2 images for our sources reveals clear signatures of compact dust lanes. As this dust and the ionized gas are most likely part of the same central disk (e.g., Pogge et al. 2000; Verdoes Kleijn et al. 1999), the dust-lane morphology provides a potential means of deriving the disk orientation. We obtained corresponding estimates of $i$ and $\phi$ for the gas disk by defining 
ellipses consistent with the dust-lane morphology and assuming that deviations from circularity result from inclination and projection. An objective ellipse-fitting algorithm for the dust features is difficult to construct, since the strength of the dust lanes varies spatially and is not continuous. We therefore constructed ellipses by eye that are consistent with the dust-lane morphology, with the outcome shown in Figure 3. These results were checked with absorption maps generated by subtraction of a smooth starlight profile from the original images. Estimates of ellipse parameters obtained independently by different members of our team (M. S. and H. W. R.) showed good agreement $\left[\Delta(b / a) \lesssim 0.1\right.$ and $\Delta$ (P.A.) $\left.\lesssim 10^{\circ}\right]$, providing some confidence in these fits. Note that all the dust rings seem quite highly inclined. In this inclination regime $\sin i$ is not very sensitive to errors in $i$. We used the results to generate a second, constrained set of models with the disk orientation specified by the dust morphology.

For a given $i$ and $\phi$ and a given total potential $\Phi(r)=$ $\Phi_{\mathrm{BH}}(r)+\Phi_{*}(r)$, the projected line-of-sight gas velocity field $V_{p}\left(x_{p}, y_{p}\right)$ is completely specified by our model. To make observable predictions, this velocity field must be further weighted by the spatial distribution of line flux and convolved by the HST PSF. Directly observed gas disks on parsec scales (e.g., M87; Harms et al. 1994) show that the gas may be patchy. Given our limited data, our best guess for the two-dimensional gas emissivity distribution $f\left(x_{p}, y_{p}\right)$ is still the axisymmetric extension of the deconvolved line flux profile along the slit. The latter was obtained also through a multi-Gaussian deprojection. The observable velocity field resulted then from convolving $f\left(x_{p}, y_{p}\right) V_{p}\left(x_{p}, y_{p}\right)$ with the PSF and sampling the result along the slit. This procedure provides model points for direct comparison with our STIS measurements.

\section{MODELING RESULTS}

\subsection{Model-Data Comparison}

In the unconstrained case, we considered all possible orientations, or the full $(i, \phi)$ parameter space. For any given black hole mass $M_{\mathrm{BH}}$ and its corresponding $\Upsilon\left(M_{\mathrm{BH}}\right)$, we identified the set of $(i, \phi)$ that best matches the observed kinematics. The quality of this match was quantified by $\chi_{\mathrm{free}}^{2}\left[M_{\mathrm{BH}} \mid \Upsilon\left(M_{\mathrm{BH}}\right)\right]$ values, each optimized over $(i, \phi)$. For fits with fixed disk orientation, we simply adopted the values from the dust-lane morphology and explored $\chi_{\mathrm{fix}}^{2}\left[M_{\mathrm{BH}} \mid \Upsilon\left(M_{\mathrm{BH}}\right)\right]$ for a fixed $(i, \phi)$. By constructing $\chi_{\text {free }}^{2}$ and $\chi_{\text {fix }}^{2}$ curves we can check more stringently the necessity for a central mass concentration, eventually deriving the best $M_{\mathrm{BH}}$ values for each case.

We considered a number of errors and modeling uncertainties and assessed their importance for the $M_{\mathrm{BH}}$ estimates through simulations. This analysis considered uncertainties in (1) the derived $v(r)$, particularly at small $r$, (2) the multiGaussian fit to the surface brightness of the ionized gas, (3) the location of the kinematical center of the observed rotation curves, and (4) $\Upsilon\left(M_{\mathrm{BH}}\right)$, including the effects of possible deviations from our assumption of sphericity and isotropy for the stellar distribution and the variance among published values for the central velocity dispersions. Neglecting the intrinsic flattening can lead us to underestimate the mass-to-light ratio $\Upsilon$; from the tensor virial theorem the fractional error on $\Upsilon$ can be evaluated to be less than half of the intrinsic axis ratio $c / a$ (Kronawitter et al. 2000). Assuming for our four galaxies a typical apparent flattening in their central regions of $b / a=0.85$ (Fig. 1) and an inclination of $40^{\circ}$ (Table 1$)$, their intrinsic axis ratios would be around $c / a=0.6$, and the mass-to-light ratio would need to be increased by $21 \%$ (Kronawitter et al. 2000). Note that the published values for the central velocity dispersion of our sample galaxies have a variance of $3 \%-20 \%$.

The uncertainty in $\Upsilon$ proved to have the largest impact on $M_{\mathrm{BH}}$. Therefore, we obtained two additional fitting sequences $\chi^{2}\left[M_{\mathrm{BH}} \mid \Upsilon\left(M_{\mathrm{BH}}\right)\right]$, adopting stellar mass-to-light ratios equal to 0.7 and 1.3 times the derived $\Upsilon\left(M_{\mathrm{BH}}\right)$, in both the unconstrained and constrained disk orientation cases. In essence, we are hereby allowing that our simple modeling has introduced $30 \%$ systematic errors in the $\Upsilon\left(M_{\mathrm{BH}}\right)$ determination.

The rotation curves in our culled sample show small wiggles and asymmetries in excess of their statistical errors, presumably because of nongravitational phenomena acting on the gas or to the patchiness of the surface brightness distribution of the gas (e.g., Barth et al. 2001). In practice, this means that we cannot expect to generate a model that is formally acceptable in a $\chi^{2}$ sense, and the estimation of confidence limits is correspondingly affected. To allow for the additional velocity structure that is not addressed by our model, we rescaled all $\chi^{2}$ values to achieve $\chi_{\text {free }}^{2}=$ $N_{\text {DOF }} \equiv N_{\text {Data }}-N_{\text {fit }}$ for the best unconstrained fit, where $N_{\text {DOF }}, N_{\text {Data }}$, and $N_{\text {fit }}$ are the numbers of degrees of freedom, data points, and fit parameters, respectively. Note that this procedure is conservative in the sense of widening the confidence intervals. Confidence limits on the range of $M_{\mathrm{BH}}$ were then derived from a $\Delta \chi^{2}$ test (e.g., Press et al. 1986), for the variation of one parameter.

\subsection{Fits with Unconstrained Disk Orientation}

The results of the unconstrained fits are shown in the top and middle panels of Figures $4 a, 4 b, 4 c$, and $4 d$ and are summarized in Table 2. The resulting uncertainties in $M_{\mathrm{BH}}$ are large, and only one of the objects (NGC 2787) exhibits evidence of a nonzero mass at more than the $2 \sigma$ level. In all sample galaxies the gas rotation curve extends beyond the radii where the black hole is likely to dominate; there the observed gas motion should approach the one predicted from the stellar mass, which was obtained from the stellar modeling. This requirement provides a constraint on the

TABLE 2

MODEL Results

\begin{tabular}{cccccc}
\hline \hline $\begin{array}{c}\text { Galaxy } \\
(1)\end{array}$ & $\begin{array}{c}M_{\mathrm{BH}, \mathrm{free}} \\
(2)\end{array}$ & $\begin{array}{c}M_{\mathrm{BH}, \mathrm{fix}} \\
(3)\end{array}$ & $\begin{array}{c}i \\
(4)\end{array}$ & $\begin{array}{c}\phi \\
(5)\end{array}$ & $\begin{array}{c}M_{\text {sph }} \\
(6)\end{array}$ \\
\hline NGC $2787 \ldots \ldots$ & $18.5_{-14.2}^{+50.8}$ & $7.1_{-0.9}^{+0.7}$ & 49 & 14 & 2.9 \\
NGC $4203 \ldots \ldots$ & $<0.5$ & 5.2 & 49 & 75 & 1.5 \\
NGC $4459 \ldots \ldots$ & $1.8_{-1.2}^{+19.2}$ & $7.3_{-1.4}^{+1.4}$ & 47 & 8 & 6.4 \\
NGC $4596 \ldots \ldots$ & $0.8_{-0.8}^{+49.3}$ & $7.8_{-3.3}^{+3.8}$ & 40 & 41 & 8.6 \\
\hline
\end{tabular}

Note.-Cols. (2) and (3): $M_{\mathrm{BH}}$ estimates or upper limits in units of $10^{7} M_{\odot}$, along with $3 \sigma$ and $1 \sigma$ confidence limits, for the unconstrained and constrained models, respectively. The only exception is the upper limit for the black hole mass of NGC 4203, which was derived according to the $3 \sigma$ confidence limit. Cols. (4) and (5): angles describing gas disk orientation, in degrees, for the constrained models. Col. (6): bulge mass in units of $10^{10} M_{\odot}$ derived from the luminosities in Table 3 and the mean $\Upsilon$ derived by van der Marel 1991. 


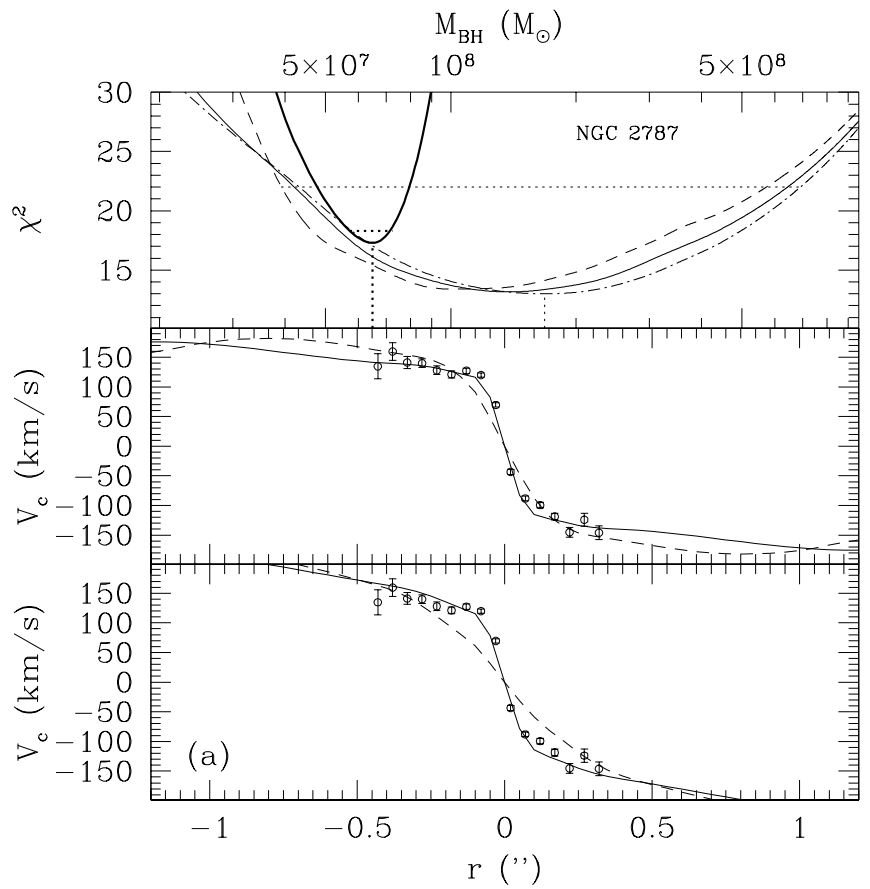

FIG. $4 a$

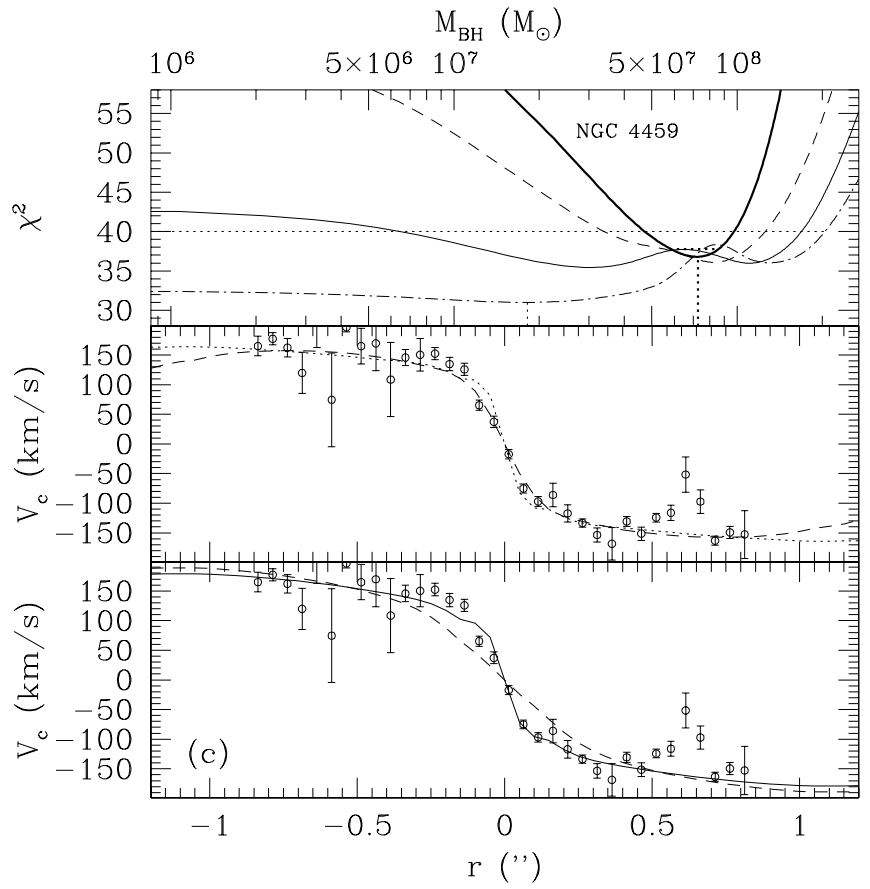

FIG. $4 c$

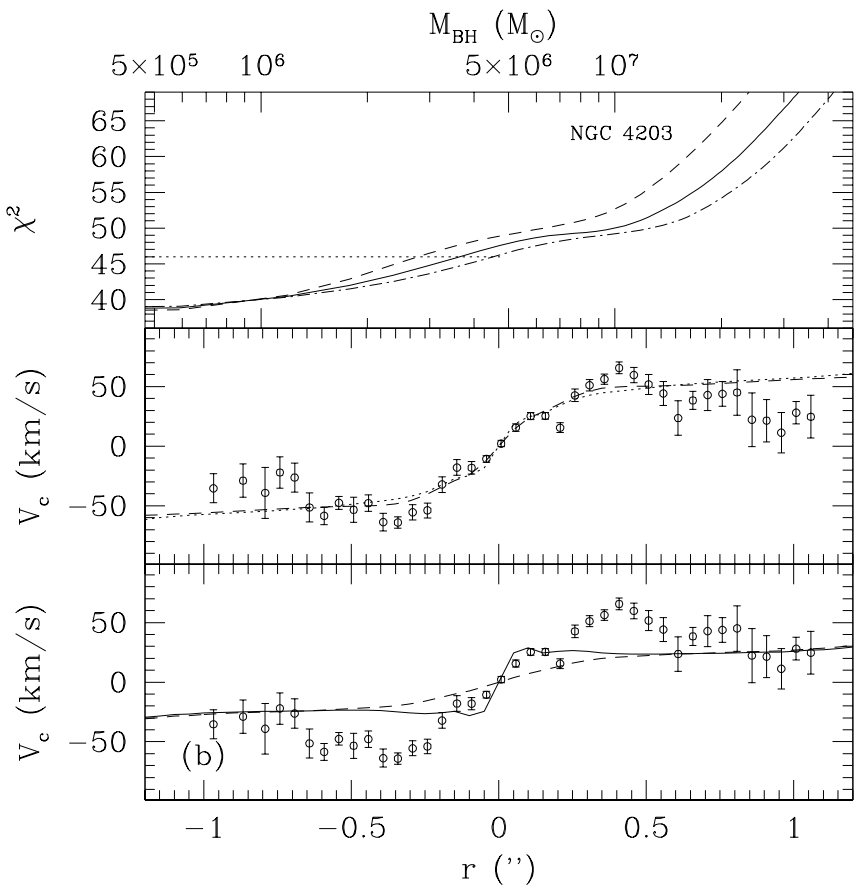

Fig. $4 b$

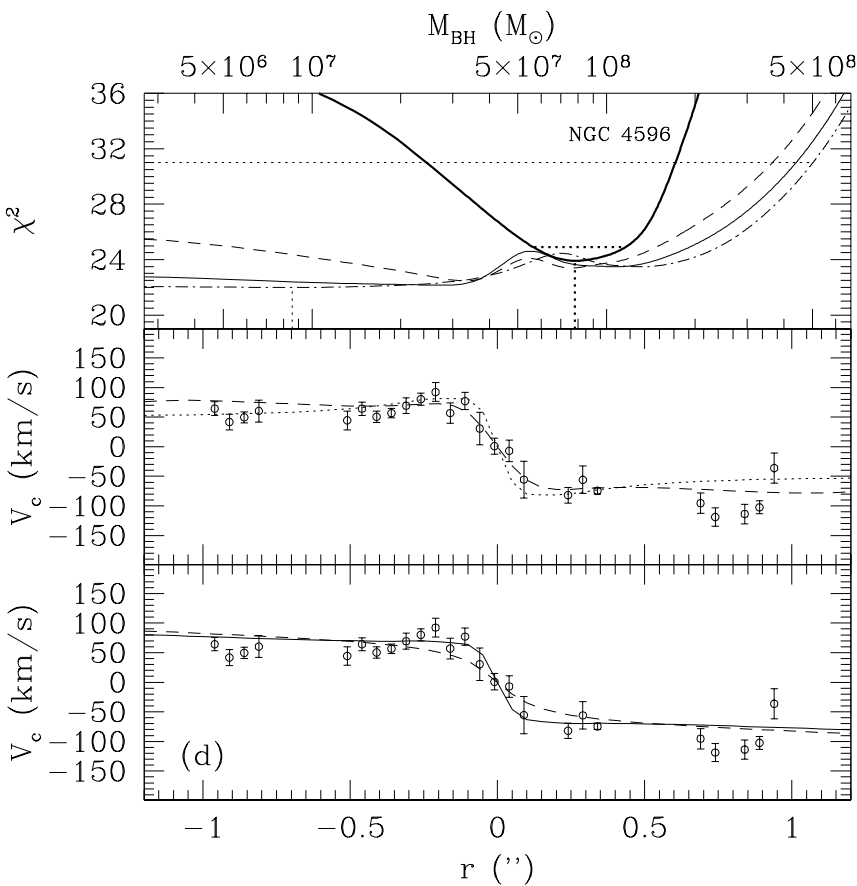

FIG. $4 d$

FIG. 4.- Observed rotation curves along with the modeling results in both the unconstrained and constrained cases. Upper panels: Rescaled $\chi_{\text {free }}^{2}($ thin lines) and $\chi_{\mathrm{fix}}^{2}$ (thick lines) as a function of $M_{\mathrm{BH}}$. The effect of adopting alternative values of $\Upsilon\left(M_{\mathrm{BH}}\right)$ rescaled by factors of $0.7,1$, and 1.3 are shown in the unconstrained case by the thin dashed, solid, and dot-dashed lines, respectively. The thin dotted lines indicate the formally best estimate of $M_{\mathrm{BH}}$ in the unconstrained case vertically and the $3 \sigma$ upper limits horizontally. The thick dotted lines mark the estimate for $M_{\mathrm{BH}}$ in the constrained case for the best $\Upsilon\left(M_{\mathrm{BH}}\right)$ fitting sequence (vertically), along with the corresponding $1 \sigma$ variances (horizontally). Middle panels: velocity as a function of position along the slit, for the observed values (points), the unconstrained model with no black hole (dashed lines), and the best-fit unconstrained model including a black hole (NGC 2787, solid lines) or $M_{\mathrm{BH}}$ set at the $3 \sigma$ upper limit (dotted lines). Lower panels: velocity as a function of position along the slit, for the observed values (points), the constrained model with no black hole (dashed lines), and the best-fit constrained model including a black hole (solid lines).

gas inclination, and precludes arbitrarily large $M_{\mathrm{BH}}$ with disks seen at large $i$. In this way, we can obtain robust upper limits on $M_{\mathrm{BH}}$, which prove interesting in some cases such as NGC $4203\left(M_{\mathrm{BH}}<5 \times 10^{6} M_{\odot}\right.$ at $\left.3 \sigma\right)$, which is dis- cussed in detail by Shields et al. (2000). We note that the limiting value presented here for this object is $20 \%$ smaller than the value reported by Shields et al. (2000); this difference is a result of the use of $\Upsilon\left(M_{\mathrm{BH}}\right)$ in the present analysis, 
rather than a mass-to-light ratio that neglects the presence of the black hole.

\subsection{Fits with Fixed Disk Orientation}

The results of the fits with constrained disk inclination are shown in the top and lower panels of Figures $4 a, 4 b, 4 c$, and $4 d$ and are reported in Table 2 . We note that in all cases except NGC 4203, the constrained fit is statistically comparable (in a $\chi^{2}$ sense) to the unconstrained fit and shows evidence for a central mass concentration at a less than $2 \sigma$ level. The quality of the fit is thus not improved by relaxing the disk orientation while maintaining $M_{\mathrm{BH}}$ at the value dictated by the minimum $\chi_{\mathrm{fix}}^{2}$. This finding is indicated in Figures $2 a, 2 c$, and $2 d$ by the fact that the thick curves representing $\chi_{\mathrm{fix}}^{2}$ coincide with the thin curves where $\chi_{\mathrm{fix}}^{2}$ attains a minimum. This result means that the unconstrained procedure leads to $(i, \phi)$ values very similar to the one derived from the dust patterns, providing strong supporting evidence that this is indeed the correct choice of orientation.

Not surprisingly, Figure 4 shows that use of the fixed disk orientation constrains $M_{\mathrm{BH}}$ much more tightly, with $M_{\mathrm{BH}} \approx 7 \times 10^{7} M_{\odot}$ for each of NGC 2787 , NGC 4459, and NGC 4596; the individual values and their uncertainties are listed in Table 2. These errors reflect the $1 \sigma$ confidence regions for $M_{\mathrm{BH}}$ from the constrained fit after considering all the three alternative values of $\Upsilon\left(M_{\mathrm{BH}}\right)$. For NGC 4459, Bertola et al. (1998) derived from ground-based observations an upper limit for $M_{\mathrm{BH}}$ of $10^{9} M_{\odot}$ with an uncertainty of a factor of 3. For NGC 4203 the best agreement was found with $M_{\mathrm{BH}}=5.2 \times 10^{7} M_{\odot}$. For this last object, the minimum $\chi_{\mathrm{fix}}^{2}$ is dramatically worse than $\chi_{\mathrm{free}}^{2}$ at the same $M_{\mathrm{BH}}$ and depends sensitively on the assumed angle $\phi$; in our constrained case, $\phi=75^{\circ}$, representing a slit P.A. near the minor axis of the disk. None of the models for this source adequately reproduces both the inner and outer parts of the rotation curve in detail. The constrained fit appears consistent at large radii $(r \gtrsim 0$.'7) but invariably shows substantial deviations in the central region that produce the large $\chi_{\mathrm{fix}}^{2}$. Since the outer disk in this model appears consistent with the orientation selected by the dust lanes, which reside at even somewhat larger radii, and taking into account that the innermost part of the rotation curve is nicely matched in the unconstrained case, we speculate that the rotation curve is affected by a warp in the gas disk in this source. Besides, gas kinematics in other galactic nuclei have been successfully modeled by taking warped structures into account (e.g., NGC 3227; Shinnerer et al. 2000). In light of these difficulties with the constrained fit, we adopt here only an upper limit on $M_{\mathrm{BH}}$ based on the unconstrained model, as reported also by Shields et al. (2000).

\section{DISCUSSION}

The results of this study are important in several ways for the understanding of SMBHs in galaxies. For all four galaxies analyzed here, we find qualitative and/or quantitative evidence for the existence of SMBHs at their centers. For three sources we obtained well-determined values of $M_{\mathrm{BH}}$; for the fourth object, NGC 4203, the likely presence of a SMBH is signaled by very broad $\left( \pm 3000 \mathrm{~km} \mathrm{~s}^{-1}\right) \mathrm{H} \alpha$ emission (Shields et al. 2000). An important point, therefore, is that all of the sources examined here apparently harbor an
$\mathrm{SMBH}$, reinforcing indications that such objects are an integral part of most galaxies. We emphasize that our four objects were selected from the SUNNS sample on the basis of the angular extent $(>0.5)$ and symmetry of their velocity curves, not on the existence of a steep central velocity gradient or other criteria indicative of an SMBH. We note also that the majority of objects with kinematic measurements of $M_{\mathrm{BH}}$ to date are elliptical galaxies (FM00; G00, and references therein), so that the present results for four S0 galaxies represent a substantial increase in the census of SMBHs in bulges of disk galaxies. The findings described here are also important for comparison with suggested trends relating $M_{\mathrm{BH}}$ to galaxy bulge properties. For our four galaxies, we obtained bulge $B$-band luminosities using $V$-band diskbulge decompositions from Baggett, Baggett, \& Anderson (1998), based on digitized photographic plates from Kodaira, Okamura, \& Ichikawa (1990) and $B-V_{e}$ colors within the galaxy effective radius, with the results listed in Table 3. Bulge masses were estimated using the mean $B$-band $\Upsilon=8.90(M / L)_{\odot}$ from van der Marel (1991), rescaled to $H_{0}=75 \mathrm{~km} \mathrm{~s}^{-1} \mathrm{Mpc}^{-1}$. For comparison with FM00 and G00, we also calculated stellar velocity dispersions $\sigma_{e}$ and $\sigma_{e / 8}$ within the bulge effective radius $R_{e}$ and within $R_{e} / 8$, respectively, using values of $\sigma$ from Table 1 , seeing-corrected measurements of $R_{e}$ from Baggett et al. (1998), and the algorithm of Jorgensen, Franx, \& Kjaegaard (1995).

Our results for $M_{\mathrm{BH}}$ as a function of bulge properties are shown in relation to previous work in Figure 5. Compared with the $M_{\text {bulge }}-M_{\mathrm{BH}}$ correlation reported by Magorrian et al. (1998), our constrained results yield black hole masses that are lower by a factor of $\sim 3$ or more at a given $M_{\text {bulge }}$ while two out of four of our unconstrained $3 \sigma$ upper limits equally fall below the $M_{\text {bulge }}-M_{\mathrm{BH}}$ relation, consistent with other suggestions that the two-integral estimates of $M_{\mathrm{BH}}$ by these authors are systematically too large (e.g., van der Marel 1998). When compared with published analyses employing more robust measures of SMBH masses, our results show better agreement, as can be seen in the diagram of $M_{\mathrm{BH}}$ versus $L_{\text {bulge }}$ from Ho (1999), which derived a $M_{\mathrm{BH}} / L_{\text {bulge }}$ ratio equivalent to a $M_{\mathrm{BH}} / M_{\text {bulge }} \simeq 0.002$, quite similar to the value first inferred by Kormendy \& Richstone (1995). The constrained fits in particular are in good agreement with the latter correlation, although the upper limit for NGC 4203 continues to suggest a nonnegligible degree of scatter in the $M_{\mathrm{BH}}$ versus $L_{\text {bulge }}$ relation.

The relationship between $M_{\mathrm{BH}}$ and $\sigma$ is of particular interest in light of recent reports by FM00 and G00 of a

TABLE 3

Bulge Properties of Selected Galaxies

\begin{tabular}{|c|c|c|c|c|c|c|}
\hline $\begin{array}{l}\text { Galaxy } \\
\text { (1) }\end{array}$ & $\begin{array}{l}V_{\text {tot }} \\
(2)\end{array}$ & $\begin{array}{c}V_{\text {bulge }} \\
\text { (3) }\end{array}$ & $\begin{array}{l}B-V_{e} \\
\text { (4) }\end{array}$ & $L_{\text {bulge }}\left(10^{9} L_{\odot}\right)$ & $\begin{array}{l}\sigma_{e} \\
(6)\end{array}$ & $\begin{array}{l}\sigma_{e / 8} \\
(7)\end{array}$ \\
\hline NGC $2787 \ldots$ & 10.76 & 11.17 & 1.10 & 3.25 & 184.9 & 206.5 \\
\hline NGC $4203 \ldots$ & 10.86 & 11.33 & 0.98 & 1.74 & 109.9 & 122.7 \\
\hline NGC $4459 \ldots$ & 10.37 & 10.99 & 0.98 & 7.16 & 166.6 & 186.1 \\
\hline NGC $4596 \ldots$ & 10.41 & 10.69 & 0.96 & 9.61 & 136.3 & 152.3 \\
\hline
\end{tabular}

Note.-Col. (2): galaxy total $V$-band magnitude from de Vaucouleurs et al. 1991. Col. (3): $V$-band magnitude for galaxy bulge light, obtained by subtracting from the total galaxy light the disk contribution inferred from Baggett et al. 1998. Col. (4): galaxy $B-V_{e}$ effective colors from de Vaucouleurs et al. 1991. Col. (5): bulge $B$-band luminosities. Cols. (6) and (7): stellar velocity dispersion $\sigma_{e}$ and $\sigma_{e / 8}$ in $\mathrm{km} \mathrm{s}^{-1}$, within a circular aperture of radius $R_{e}$ or $R_{e / 8}$, respectively. 


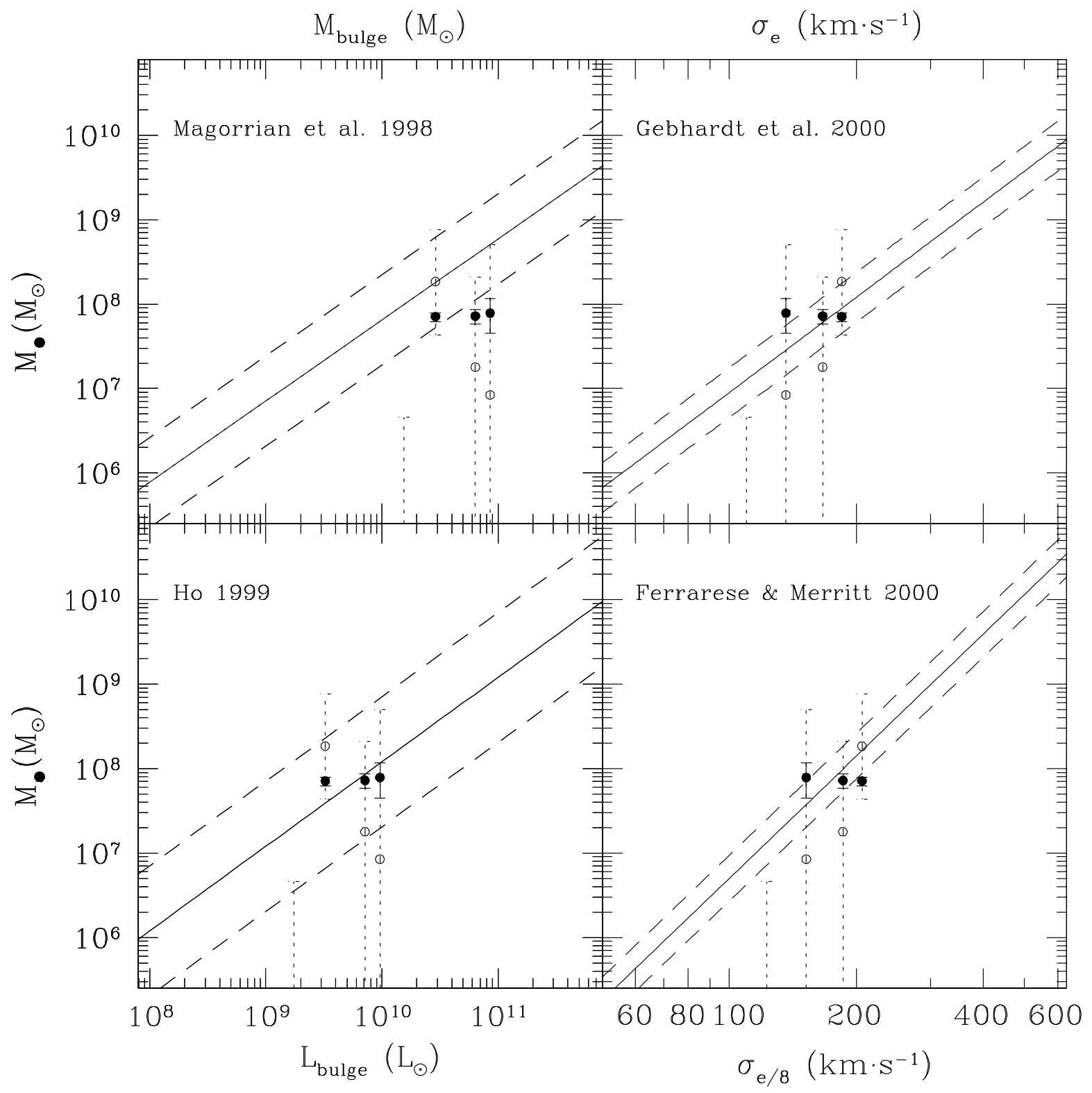

FIG. 5. $-M_{\mathrm{BH}}$ vs. $M_{\text {bulge }}$ and $L_{\text {bulge }}$ (left panels) and vs. $\sigma_{e}$ and $\sigma_{e / 8}$ (right panels). The solid diagonal lines represent the correlation fits reported previously by the indicated authors, with dashed diagonal lines representing the $1 \sigma$ scatter in $M_{\mathrm{BH}}$. Best black hole mass estimates or upper limits are shown for models with disk unconstrained orientation (open circles, dashed limits) and constrained orientation (filled circles, solid limits). Error bars correspond to $1 \sigma$ and $3 \sigma$ confidence limits, in the constrained and unconstrained case, respectively.

very significant correlation with little intrinsic scatter. Estimates of stellar velocity dispersion employed by these authors correspond to apertures large enough to avoid the influence of a black hole on $\sigma$ itself. While our data considered in isolation show no correlation between $M_{\text {bulge }}$ and any of the other quantities considered so far, it is worth noticing that while going from the unconstrained to the constrained procedure, the black hole mass determinations move closer to the $M_{\mathrm{BH}}$ versus $L_{\text {bulge }}, \sigma_{e}$, and $\sigma_{e / 8}$ relations. Our results, and the constrained fits in particular, are in good agreement with the quantitative relations given in both papers. These findings provide added support for the strength of an $M_{\mathrm{BH}^{-}} \sigma$ trend, and its persistence in bulges associated with disk galaxies.

As a final comment, we note that while the present work provides an illustration of the power of gas kinematics for finding and quantifying the masses of SMBHs, it also shows that a significant investment of observing time and effort is necessary in order to yield useful results. In particular, the SUNNS survey demonstrates that only a modest fraction $(\$ 25 \%)$ of nearby galaxies displaying emission-line nuclei in ground-based observations can be expected with $H S T$ to show spatially resolved nebular emission with regular kinematics suitable for estimation of $M_{\mathrm{BH}}$. Determination of the gas velocity field with high confidence requires twodimensional mapping with multiple slit positions (e.g., Barth et al. 2001), which is expensive in terms of telescope time. The alternative of long-slit observations at a single P.A. can be employed with some effort to extract useful estimates of $M_{\mathrm{BH}}$, as demonstrated by the present work. In particular, the analysis reported here further illustrates the power of using dust morphology as an indicator of gas disk 
orientation, in the absence of full two-dimensional velocity data.

This research was supported financially through NASA grant NAG 5-3556 and by GO-07361-96A, awarded by
STScI, which is operated by AURA, Inc., for NASA under contract NAS 5-26555. M. Sarzi gratefully acknowledges N. Cretton and W. Dehnen for valuable comments and suggestions on this work.
Baggett, W. E., Baggett, S. M., \& Anderson, K. S. J. 1998, AJ, 116, 1626

Barth, A. J., Sarzi, M., Rix, H.-W., Ho, L. C., Filippenko, A. V., \& Sargent, W. L. W. 2001, ApJ, submitted (astro-ph/0012213)

Bertola, F., Cappellari, M., Funes, J., Corsini, E. M., Pizzella, A., \& Vega Beltrán, J. C. 1998, ApJ, 509, L93

Binney, J., \& Tremaine, S. 1987, Galactic Dynamics (Princeton: Princeton Univ. Press)

Dalle Ore, C., Faber, S. M., Gonzalez, J. J., Stoughton, R., \& Burstein, D. 1991, ApJ, 366, 38

de Vaucouleurs, G., de Vaucouleurs, A., Corwin, H. G. Jr., Buta, R. J., Paturel, G., \& Fouqué, P. 1991, Third Reference Catalogue of Bright Galaxies (New York: Springer) (RC3)

Ferrarese, L., \& Merritt, D. 2000, ApJ, 539, L9 (FM00)

Filippenko, A. V., \& Sargent, W. L. W. 1985, ApJS, 57, 503

Gebhardt, K., et al. 2000a, ApJ, 539, L13 (G00) . 2000b, ApJ, 534, 25

Harms, R. J., et al. 1994, ApJ, 435, L35

Hernquist, L. 1990, ApJ, 356, 359

Ho, L. 1999, in Observational Evidence for the Black Holes in the Universe, ed. S. K. Chakrabarti (Dordrecht: Kluwer), 157

Ho, L. C., Filippenko, A. V., \& Sargent, W. L. W. 1997a, ApJS, 112, 315

Ho, L. C., Filippenko, A. V., Sargent, W. L. W., \& Peng, C. Y. 1997b, ApJS, 112,391

Jorgensen, I., Franx, M., \& Kjaergaard, P. 1995, MNRAS, 276, 1341

Kaspi, S., Smith, P. S., Netzer, H., Maoz, D., Jannuzi, B. T., \& Giveon, U. 2000, ApJ, 533, 631

Kauffmann, G., \& Haehnelt, M. 2000, MNRAS, 311, 576

Kent, S. M. 1990, AJ, 100, 377

Kodaira, K., Okamura, S., \& Ichikawa, S., eds. 1990, Photometric Atlas of Northern Galaxies (Tokyo: Univ. Tokyo Press)

Kormendy, J. 1993, in The Nearest Active Galaxies, ed. J. Beckman, L. Colina, \& H. Netzer (Madrid: CSIC Press), 197

\section{RFERENCES}

Kormendy, J., \& Richstone, D. 1995, ARA\&A, 33, 581

Kriss, G. 1994, in ASP Conf. Ser. 61, Astronomical Data Analysis Software and Systems III, ed. D. R. Crabtree, R. J. Hanisch, \& J. Barnes (San Francisco: ASP), 437

Krist, J., \& Hook, R. 1999, The Tiny Tim User's Guide (Baltimore: STScI)

Kronawitter, A., Saglia, R. P., Gerhard, O., \& Bender, R. 2000, A\&AS, 144, 53

Magorrian, J., et al. 1998, AJ, 115, 2285

Monnet, G., Bacon, R., \& Emsellen, E. 1992, A\&A, 253, 366

Nagar, N. M., Falcke, H., Wilson, A. S., \& Ho, L. C. 2000, ApJ, 543, 136

Narayan, R., Mahadevan, R., \& Quataert, E. 1998, in Theory of Black Hole Accretion Disks, ed. M. A. Abramowicz, G. Bjornsson, \& J. E. Pringle (Cambridge: Cambridge Univ. Press), 148

Norman, C. A., Sellwood, J. A., \& Hasan, H. 1996, ApJ, 462, 114

Pogge, R. W., Maoz, D., Ho, L. C., \& Eracleous, M. 2000, ApJ, 532, 323

Press, W. H., Flannery, B. P., Teukolsky, S. A., \& Vettering, W. T. 1986, Numerical Recipes: The Art of Scientific Computing (Cambridge: Cambridge Univ. Press)

Schinnerer, E., Eckart, A., \& Tacconi, L. J. 2000, ApJ, 533, 826

Shields, J. C., Rix, H.-W., McIntosh, D. H., Rudnick, G., Ho, L. C., Filippenko, A. V., Sargent, W. L. W., \& Sarzi, M. 2000, ApJ, 534, L27

Terashima, Y., Ho, L. C., \& Ptak, A. F. 2000, ApJ, 539, 161

Tully, R. B. 1988, Nearby Galaxies Catalog (Cambridge: Cambridge Univ. Press)

van der Marel, R. P. 1991, MNRAS, 253, 710 1998, in IAU Symp. 186, Galaxy Interactions at Low and High Redshift, ed. D. B. Sanders \& J. Barnes (Dordrecht: Kluwer), 333

Verdoes Kleijn, G. A., Baum, S. A., de Zeeuw, P. T., \& O'Dea, C. P. 1999, AJ, 118, 2592

Wandel, A. 1999, ApJ, 519, L39 